\title{
Hearing aids: recommendations, developments and practicalities
}

Keywords: amplifier products, Consumer, hearing aids, hearing, listening

Abbreviations: PSAPs, personal sound amplifier products; DTC, direct to consumer; HCPs, hearing care professionals; SIN, speech in noise

\section{Introduction}

As hearing aid dispensing increasingly steps into the mainstream, multiple consumer look-alike products and alternatives evolve such as personal sound amplifier products (PSAPs) and the soon-to-be-FDAapproved Over The Counter (OTC) hearing aid products. ${ }^{1}$ Further, big box hearing aid dispensing operations such as Costco and Sam's Club, in addition to Direct to Consumer (DTC) products sold via the mail and Internet, also garner attention. As such, many people query, "what's the difference?" Hearing Care Professionals (HCPs) and consumers want to know whether the least expensive products are just as good as the premium products? Are specific features better than others? Where is the best place to acquire hearing aids? Are all hearing aids the same? How should these products be programmed? These are very important questions. In this article, we'll review some of the most important concepts and ideas, and we'll offer some insight and practical advice based on our decades of experience as audiologists, doctors and researchers.

\section{Hearing and/or listening problems}

"Hearing" is simply the ability to detect sound, whereas "listening" is the ability to untangle, make sense of, apply meaning to, or comprehend sound. These distinctions are important as the most common complaint across the majority of patients with demonstrable hearing loss is the inability to understand speech in noise (SIN). In the USA, there are approximately 37 million people with hearing loss measurable on an audiogram. Of equal importance, there are an additional 26 million people in the USA without measurable hearing loss on an audiogram who have hearing difficulty (HD) or SIN problems. ${ }^{2,3}$ Many animals have 'super-human' hearing; they can hear softer, lower pitched, and/or higher pitched sounds, than humans can. For example, rats can perceive sounds to $80,000 \mathrm{~Hz}$, house cats often hear to $60,000 \mathrm{~Hz}$, dogs may hear up to $45,000 \mathrm{~Hz}{ }^{4}$

However, humans are the "top of the food chain" not due to our relatively insensitive hearing, but due to our ability to listen; to apply meaning to sound (i.e., to develop and use language etc.). Thus, although one must certainly "hear" before one can "listen," hearing is not the end-game. Listening is. Indeed, "Listening is where hearing meets brain".

\section{Audiograms}

Importantly, audiograms do not measure "listening" they measure "hearing" by plotting loudness versus frequency. Yet audiograms only reveal hearing thresholds (i.e., how loud a sound must be to be audible and perceived $50 \%$ of the time) across specific frequencies (250 to $8000 \mathrm{~Hz}$ ). The stimuli used to acquire audiograms are pure-tones.
Volume II Issue 6 - 2019

\author{
Douglas L Beck,' John Pumford, ${ }^{2}$ Meng Guo \\ 'Executive Director of Academic Sciences, Oticon Inc., USA \\ ${ }^{2}$ Director of Audiology and Education,Audioscan, Canada \\ ${ }^{3}$ Research Engineer, Discovery Demant, Denmark
}

Correspondence: Douglas L Beck, Executive Director of Academic Sciences, Oticon Inc, Somerset, NJ, USA, Emaildbec@oticon.com

Received: September 13, 2019 | Published: November 25, 2019

Although pure-tone thresholds are very useful to determine typical highly recognized audiometric patterns (otosclerosis, presbycusis, noise induced hearing loss and more) it is important to realize that the primary signal of interest in the real world is speech, not puretones. Additionally, an audiogram doesn't measure all (or even most) of human hearing. The lowest tone tested on an audiogram is $250 \mathrm{~Hz}$, which corresponds approximately to middle " $\mathrm{C}$ " on a piano. Therefore, the entire lower left side of the piano is not reflected on an audiogram. Timmer, Hickson and Launer ${ }^{6}$ reported a "weak correlation" between pure tone averages (PTAs) and self-reported hearing difficulties. Beck \& Danhauer ${ }^{2}$ and Beck, Danhauer \& Abrams et al., ${ }^{3}$ report 26 million Americans have hearing difficulty and/or SIN problems in the presence of normal pure tone thresholds. Shub, Makashay \& Brungart ${ }^{7}$ reported on more than 5000 people with "hearing critical tasks." Their results stated audiograms are a "relatively insensitive predictor of performance" on SIN tasks. Further, to improve one's performance on SIN tasks, the primary factor is not simply or solely improving audible sounds (i.e., normalizing the audiogram). Rather, as Goyette, Crukley \& Glaster ${ }^{8}$ reported, the primary factor is providing an improved signal-to-noise ratio (SNR).

\section{Recorded stimuli speech audiometry}

Specifically, many HCPs measure the ability to repeat words using a simple list of words presented in quiet. These words-in-quiet are referred to commonly as "word discrimination lists" or "word recognition lists." However, there are many problems with these word lists. First, although they are available as recorded digital presentations which are replicable and calibrated, most HCPs present "live voice" versions of the same. There are multiple problems with live voice presentations including their lack of repeatability and associated inaccuracy versus recorded presentations. That is, although the HCP may attempt to present each word list at roughly the same loudness as determined by a live-voice root-mean-square (RMS) based VU meter, the live voice presentation does not account for variability in room noise where the microphone is located, length of utterance, time between presentations, accents (of the listener and the talker), peakto-peak amplitude variability, male versus female voices and more. With regard to speech audiometry and correlations with pure tones, 
the Speech Reception threshold (SRT) is a weak measure as it, too, measures the $50 \%$ threshold level at which spondaic words can be repeated. However, there are many exceptions to the rules based on the 2 or 3 or 4 frequency pure tone average, and again, live voice presentations are common and may over-or-under represent recorded digital presentations of the same.

\section{The chief complaint}

As the primary (chief) complaint across all people with hearing and listening problems is the inability to understand speech in noise (SIN), it is extremely important to diagnose the specific problem prior to attempting to manage it. Diagnosis first, treatment second. For example, people who may have listening disorders in the presence of normal audiometric thresholds includes those with auditory processing disorders (APD), spatial hearing disorders, attention deficit disorders (ADD), dyslexia, attention deficit hyperactivity disorders (ADHD), blast exposure, traumatic brain injury (TBI), asymmetric high frequency hearing loss, neurocognitive disorders, Alzheimer's Disease, Hidden Hearing Loss, Cochlear Synaptopathy, Auditory Neuropathy Spectrum Disorder, Specific Language Impairment and more. People with these (and other) listening disorders will generally report "hearing problems" which are exacerbated in the presence of background noise. Indeed, 26 million people (USA) may experience "hearing loss" despite normal thresholds ${ }^{2,3}$ secondary to listening problems such as those noted above. Unfortunately, as sensorineural hearing loss (SNHL) progresses from normal, to mild, to moderate, to severe, to profound SNHL, simply making things louder won't necessarily make them clearer. Most audiometric cases of mildto-moderate hearing loss involve outer hair cell loss, which may benefit from a simple and appropriate loudness solution. However, as SNHL progresses past 50-65 dB HL, inner hair cells become involved, and substantial loss of clarity, sharpness, distinctness is reported, as is distortion, and a decreased dynamic range. Thus, many products which are designed essentially to make sounds louder (certain OTCs, PSAPs, DTC devices, etc.) may help resolve a simple loudness problem for some people, but for others, these approaches are inappropriate, frustrating and expensive, as the chief complaint (CC) for most patients is the inability to understand SIN; a listening problem.

As such, it is our recommendation that every individual who complains of hearing loss, hearing problems, SIN difficulties, listening disorders and related difficulties, should receive a thorough, professional, pre-and-post treatment comprehensive audiometric evaluation. The American Speech Language Hearing Association, ${ }^{9}$ the American Academy of Audiology, ${ }^{10}$ the International Hearing Society, ${ }^{11}$ and the British Society of Audiology ${ }^{12}$ note comprehensive audiometric evaluations should include a listening and/or communication assessment, Speech in Quiet (SIQ) measures, SIN measures, verification of fitted hearing aids via real-ear-probemicrophone measurements and validation measures to document patient outcomes. That is, approaching hearing loss exclusively through the lens of a pure-tone audiogram-based "one size fits all approach" (i.e., make sounds louder) may prove to be expensive, frustrating, inaccurate, inappropriate and potentially damaging.

\section{The signal-to-noise ratio (SNR)}

Of all the things we can do to help people hear and listen, the single most important thing is to improve the signal-to-noise ratio (SNR). ${ }^{2,3,8}$ The concept is to deliver the highest quality primary signal (the speech sound of interest) to the listener at a substantially louder level than other sounds (speech babble and other background noise). Of note, adults with relatively normal hearing and cognitive abilities generally require a 2-3 dB SNR to correctly repeat half the words they detect correctly. ${ }^{13}$ This metric (the SNR required to repeat $50 \%$ of the words correctly) is referred to as the "SNR-50." To listen comfortably without struggling and without substantial listening effort, a substantially higher SNR is preferred. For adults, an SNR of $10-15 \mathrm{~dB}$ is vastly preferred over an SNR of 2-3 dB. People with a mild-to-moderate SNHL may typically present with an SNR-50 of $8 \mathrm{~dB}$. Pragmatically, this means that for many people, amplifying sound, without substantially improving the SNR, only makes the sounds louder, not clearer. Again, there is more to comprehending sound than loudness.

Further, as hearing loss increases, the SNR required to understand speech (i.e., to listen clearly) increases, too. Dillon ${ }^{14}$ reported that for every $10 \mathrm{~dB}$ of hearing loss the required SNR increases $1-3 \mathrm{~dB}$. For example, the range of $0-25 \mathrm{~dB} \mathrm{HL}$ is often considered to be within normal limits for pure tone thresholds. However, given a hearing loss of $35 \mathrm{~dB}$, an appropriate amplification algorithm (for example NALNL2) should provide prescriptive loudness to "correct" the hearing loss via appropriate gain, but should also improve the SNR (at the tympanic membrane) by $3 \mathrm{~dB}$. Given a hearing loss of $45 \mathrm{~dB}$, the prescriptive algorithm provides appropriate gain to "correct" the hearing loss, but the SNR required to hear clearly in challenging situations would be 6 $\mathrm{dB}$. A hearing loss of $55 \mathrm{~dB}$ would need $9 \mathrm{~dB}$ of improved SNR and so on. Amplifying sound, in the absence of assessing and measuring individual SNR-50s, can be highly problematic and is contrary to multiple Best Practice guidelines. . $^{910,11,12}$

For adults with an SNR-50 of 1, 2 or $3 \mathrm{~dB}$ (essentially within normal limits) with mild or moderate hearing loss, they may report (or possess) no hearing or listening problems. Importantly, that doesn't mean they are "in denial." Indeed, they may be functioning at a very high level and are able to maximally use the auditory cues (such as interaural loudness differences, interaural timing differences, binaural summation, binaural squelch and more) they perceive to comprehend and untangle and understand the sounds around them. Likewise, for adults with an SNR-50 of 8,10 or $12 \mathrm{~dB}$, without audiometric hearing loss, they likely have a substantial listening problem, which should be evaluated, documented, diagnosed and managed. In summary, the SNR listening ability is as important as the pure tone thresholds to represent how an individual performs in the real world with and without amplification.

There are many excellent and innovative SIN tests commercially available in 2019. For example; The Words in Noise (WIN) Test, ${ }^{15}$ the Bamford, Kowal-Bench ${ }^{16}$ test and the relatively new (2019) Beck \& Benitez" "Two Minute Speech in Noise Test." We recommend that each patient (with or without a pure-tone hearing loss) should undergo a SIN test to determine their un-aided "baseline" SNR-50. The test is then repeated (using novel stimuli) to determine their "aided" SNR-50. In essence, the goal is to improve the SNR-50 as much as possible through hearing aid fittings, digital remote microphones, FM systems, assistive listening devices, T-coils and more, based on the specific needs of the patient as determined through communication assessments, listening assessments, case history, interview, audiograms and more.

\section{Considerations for hearing device fittings}

As hearing instrument technology evolves to address the specific 
needs of individuals with hearing and listening difficulty, vast and important questions arise regarding appropriate methods for assessing patients, fitting instruments, as well as verifying and validating outcomes. Special features such as directional microphones, noise reduction systems, processing algorithms, feedback management, wireless streaming and more, are available in sophisticated hearing aid systems to maximize outcomes for the individual. As such, programming decisions are significant as even the most basic gain, output and compression decisions (across frequencies) impact the overall hearing and listening benefit. As hearing devices have become increasingly more complicated regarding their sound processing and automatic protocols, the need for programming guidance has increased. Indeed, in sophisticated hearing devices, it is common for the $\mathrm{HCP}$ to program gain, output and compression ratios uniquely in multiple processing channels across frequencies and for a range of input loudness levels (soft, average and loud). Given the vast number of possible parameter settings, it is essential that the HCP uses clearly defined fitting rationales and decision-making approaches to maximally meet the needs of each individual patient.

Over several decades, a variety of target fitting formulas have been developed to guide the clinician in adjusting hearing aid parameters. Based on fitting goals that have been developed through research and/or consideration of hearing aid performance characteristics, these rules provide the clinician with objective targets that can be displayed in fitting software (Figure 1) and/or on separate hearing aid measurement equipment (Figure 2). These targets generally specify the gain or output desirable at various audiometric frequencies for various input levels at the eardrum of the patient.

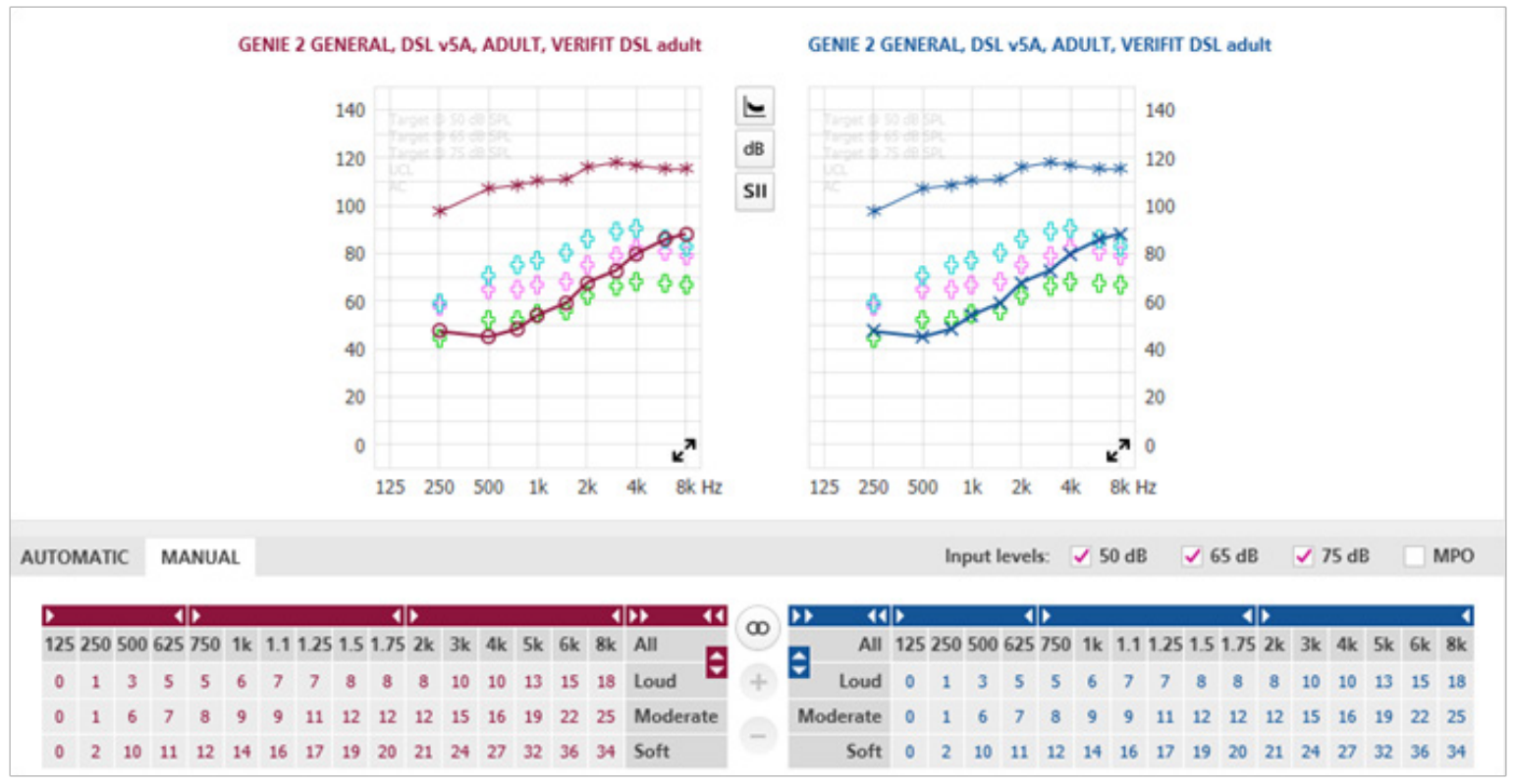

Figure I Hearing aid fitting software screen showing prescribed output targets across frequencies for multiple input levels (+'s), patient's thresholds (X's and O's) and uncomfortable loudness levels (*'s). Gain controls for various input levels across frequencies are provided in the tables to allow device fine-tuning to target and/or to patient listening preferences.

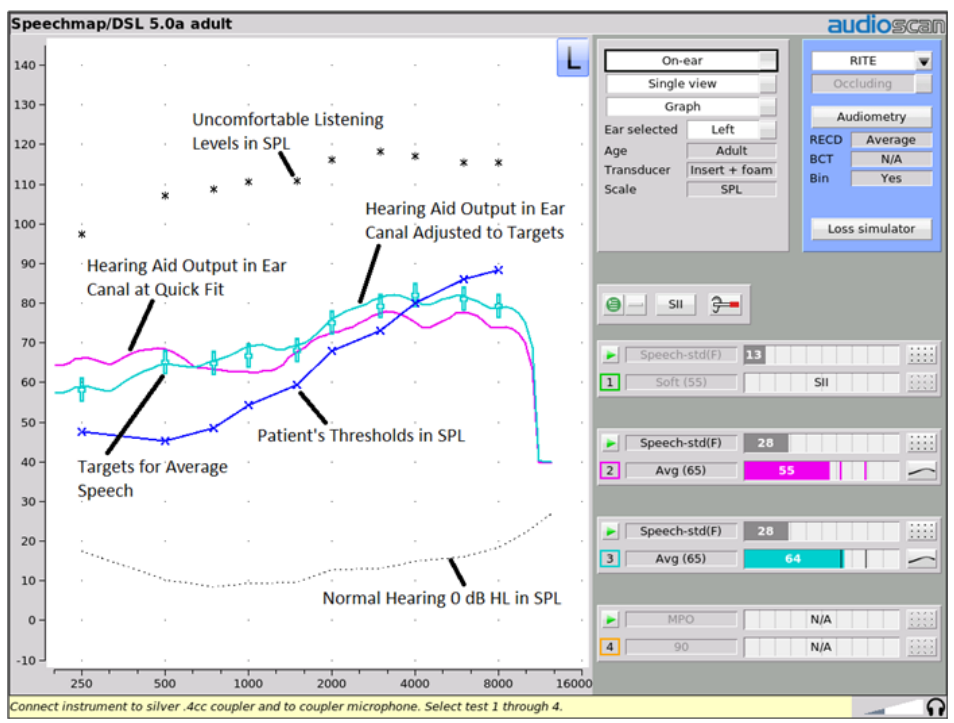

Figure 2 Hearing aid measurements conducted in ear canal of patient (Real-ear aided responses (REAR)) for average (medium level) speech. The purple curve represents the default, quick fit REAR, whereas the blue curve shows the REAR after adjustment to the prescribed DSLv5 adult targets (+'s) for average speech. Also shown are the patient's thresholds (X's), predicted uncomfortable listening levels (*'s), and normal hearing reference across frequencies, plotted in $\mathrm{dB}$ SPL at the eardrum. 


\section{Prescriptive hearing aid fitting formulas}

Prescriptive hearing aid fitting formulas provide guidance around hearing aid settings. The two most popular fitting formulas are Desired Sensation Level, ${ }^{18}$ and National Acoustic Laboratories Non-linear 2. ${ }^{19}$ The DSL method evolved from a primary focus on the listening needs of pediatric patients with an emphasis on the audibility required to develop speech and language, whereas the NAL method primarily considered the listening needs of adults with a relatively greater focus on listening comfort. Over time and with the benefit of additional research, the two fitting methods, have become increasingly similar in their prescriptions, particularly with flatter, milder hearing losses. Each method has sought to capture variables that influence the optimal sensation level of sound for a given patient across the life span. These factors include, but are not limited to: 1) Age and etiology of hearing loss (DSL v5 generally recommends more gain for pediatric patients with congenital losses than for adults with acquired losses; whereas NAL-NL2 generally provides more gain for low inputs and higher compression ratios for children than for adults); 2) Listening experience (NAL-NL2 provides additional gain for experienced hearing aid users, which differs as a function of hearing loss; whereas DSL v5 provides no correction for experience level); 3) Gender (NAL-NL2 provides $2 \mathrm{~dB}$ additional gain for male users versus female users for the same hearing loss; whereas DSL v5 provides no correction for gender); and 4) Bilateral fittings (NALNL2 provides a bilateral gain correction that increases with input level (from $2 \mathrm{~dB}$ up to $6 \mathrm{~dB}$ ) to account for binaural summation and decreases this correction with asymmetric fittings; whereas DSL $\mathrm{v} 5$ provides a $3 \mathrm{~dB}$ gain reduction for all input levels with bilateral fittings versus monaural fittings). In general, prescriptive methods will provide an audible, clear and comfortable amplified signal that ensures quality access to environmental sounds and represents an appropriate starting point for parameter settings. ${ }^{20-22}$

The peer reviewed literature demonstrates clear evidence that patients prefer devices set to validated fitting formulas and generally perform better when fitted to their prescribed targets. For instance, research with trainable hearing aids ${ }^{23,24}$ revealed that hearing aid users fitted to NAL-NL1 or NAL-NL2 on average set their use gain very similar to these prescriptions, regardless of experience with amplification. Studies evaluating DSLv5 have similarly found that patients prefer gain settings that correspond with the recommended prescription with both pediatric ${ }^{18}$ and adult patients. ${ }^{25}$ Patients overwhelmingly prefer devices verified to prescriptive targets versus unverified fittings when given the opportunity to listen to the same device set both ways. ${ }^{26,27}$ While the selection of an appropriate fitting formula as the foundation for hearing instrument parameters is important, so too, is an objective confirmation that the selected setting resulted in the desired output level at the eardrum of the patient. It is understood that there is significant variability in ear canal acoustics across patients that can significantly impact the level of amplified sound delivered to the eardrum for the exact same device settings. Factors such as the ear canal volume of the patient, the depth of insertion of the instrument, middle ear impedance, device venting characteristics are all "known unknowns" (i.e., sources of unpredictable variation and error) which can lead to varied and unpredictable real-ear aided response anomalies for the same device settings across patients.

Hearing instrument manufacturers aim to provide an initial fitting that is appropriate and delivers the prescribed amplified level at the eardrum of the patient based on the available data at hand. Yet, in this regard, there are limits to what can realistically be captured in the fitting software to "prescribe" the "first fit" of a device. While manufacturers incorporate numerous patient specific (e.g., audiometric data, ear canal acoustic data, demographic data) and device specific variables (e.g., venting parameters, microphone location effects, recommended ear coupling system, microphone calibration data), the overall combined impact of these factors on the loudness level of sound delivered to the specific individual eardrum are extraordinarily difficult to predict. In this respect, hearing aid verification measurements are the only way to be certain the device is delivering the requested output levels to the eardrum and that the goals of the selected fitting formula have been achieved. That is, although a patient's subjective report of "liking" a sound is important, it is by no means a verified, validated, scientifically acceptable, or clinically acceptable protocol to fit hearing aids, and as mentioned above, the Best Practices protocols from each of the major organizations clearly state real ear measures comprise a substantial part of clinical Best Practices. ${ }^{9,10-12}$

Verification of hearing instrument fittings generally refers to a method of objectively confirming that the hearing aid is meeting the prescribed target gain and output across frequencies for multiple input levels in the ear canal of the patient. That is, while verification can also entail objective measurements that evaluate the performance of hearing aid advanced features (e.g., noise reduction, directional microphone technology) to inform quality control, programming decisions or counseling, the primary focus remains on ensuring appropriate audibility is provided via an assessment of the match to prescriptive targets. Real-ear measurements (REM) are recommended as the preferred method of fitting and verifying hearing instruments in the best practice guidelines of numerous national and international professional bodies. ${ }^{9,10-12}$

As stated by AAA 10 "Prescribed gain (output) from a validated prescriptive method should be verified using a probe microphone approach that is referenced to ear canal SPL." Contemporary evidence demonstrates improved REM instrumentation which makes these procedures quicker and easier than ever before. For instance, automated real-ear measurement systems that are integrated within hearing aid fitting software have been shown to provide a verified fitting to prescriptive targets equivalent to manual fitting methods in roughly half the time. ${ }^{28}$ Further, new software tools are available to assist with key procedural components such as patient positioning and probe tube placement to increase the likelihood that measurements are an accurate representation of what is being delivered to the patient's eardrum. ${ }^{29}$ The reader interested in learning more about procedural details and considerations for accurate real-ear measurements is referred to Pumford and Sinclair ${ }^{30}$ and Pumford and Smriga (2018). ${ }^{31}$

The reasoning behind Best Practice recommendations for hearing instrument verification via real-ear measurement are numerous. It has been well established that hearing instruments often do not deliver what is anticipated relative to fitting formula targets upon first fit, ${ }^{32-34}$ and the device output displayed in programming software does not typically convey exactly what is actually being delivered in the ear canal..$^{35}$ These differences are common, range from small to large, are unpredictable and are mostly significant. Amlani et al., ${ }^{36}$ reported the differences between predicted and actual measures may be $10 \mathrm{~dB}$ or more. There are also multiple research studies that have documented the benefits to be achieved in hearing outcomes for patients wearing a verified hearing aid adjusted to fitting formula targets versus unverified first fits in lab-based and real-world environments..$^{26,27,37}$ Further, research has shown the potential benefits for a business' bottom line via the provision of REM services as a standard of care, 
including reduced number of office visits, ${ }^{38}$ increased loyalty to the practice, ${ }^{21,39}$ increased patient satisfaction and a willingness to pay more for clinical services. ${ }^{39}$ Summarily, there is overwhelming evidence that hearing instrument verification via REM improves the maximal hearing healthcare experience for patients and clinicians and increases the likelihood that the 'hearing improvement promises' of new hearing aid technologies are realized.

\section{How sophisticated hearing aids work in quiet and noise}

An electroacoustic phenomenon called feedback often limits the usable gain in hearing aids. Feedback problems occur when the microphone of an audio system picks-up the output from the loudspeaker/receiver, and re-amplifies sound, such that an acoustic feedback loop is created, and perceived as an annoying whistle. Although advanced techniques exist and are often used in modern hearing aids to prevent acoustic feedback ${ }^{40-44}$ the simplest and perhaps most widely used feedback prevention approach is to reduce the gain/ amplification. Obviously, gain reduction can be simply and effectively performed by patients who have a volume control by simply turning down the volume. Gain reduction can also be accomplished by HCPs during fitting sessions by lowering the prescribed gain, which might inadvertently occur secondary to engaging a "feedback manager" setting in the software. Automatic and sophisticated automatic gain reduction methods can be carried out based on the detection of system instability in hearing aids. ${ }^{45,46}$ However, the gain reduction methods noted above generally result in less-than-prescribed amplification, secondary to gain reductions. Gain reductions may suppress crucial speech cues which the brain requires to make sense of sound (i.e. successful listening), thereby potentially leading to a reduction in speech understanding, as well as an increase in listening effort, decreased satisfaction, increased returned instruments and more office visits.

Therefore, a new feedback prevention technology using spectraltemporal modulation (STM) allows the hearing aid system to deliver more gain without increasing the risk of feedback. ${ }^{47,48}$ STM processing is deployed in the time-frequency regions in which the traditional feedback control system is insufficient to remove feedback secondary to dynamic changes, such as might occur while chewing, speaking, or by moving a phone up to and around the ear. More specifically, STM processing is based upon fast feedback detection, which prevents acoustic feedback from building up, such that the hearing aid system remains stable. While using the STM system approach to feedback prevention (as demonstrated above, Figures 3-5) up to 6 $\mathrm{dB}$ more target gain is engaged - as compared to traditional hearing aid systems. ${ }^{49}$ That is, the STM approach to feedback prevention facilitates a better match to target gain during fitting procedures and provides optimal gain in dynamic situations during daily use, thus increasing the amount of audible sound (as measured by the Speech Intelligibility Index, SII) without audible feedback. ${ }^{50}$

It is now possible to amplify speech with up to $6 \mathrm{~dB}$ more gain, using STM based protocols and technology, as can be found in Oticon's Opn S and OpenSound Optimizer. Resultantly, speech understanding is improved by up to $15 \%$, listening effort may be reduced $10 \%$ (as measured via pupillometry) and memory recall is also improved $10 \% .^{51}$ Although gain reduction can lead to sound quality degradation, ${ }^{52}$ the new STM-based feedback control system in Oticon Opn S can better preserve prescribed gain, can improve speech understanding, can reduce listening effort, and can improve sound quality.

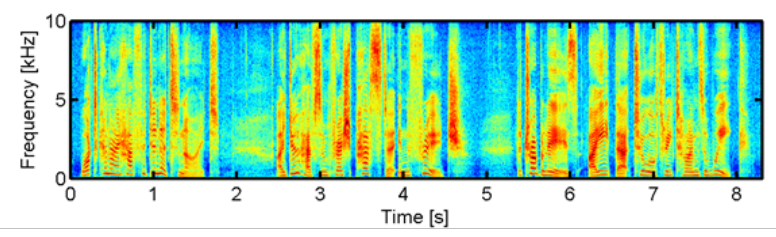

Figure 3 Spectrogram of clean speech without feedback as reference (darker colors indicate greater signal strength).

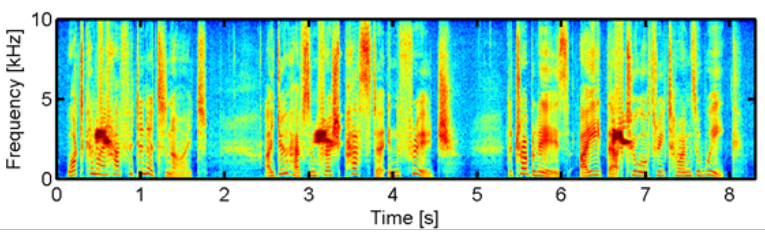

Figure 4 This spectrogram represents a traditional hearing aid feedback control system which becomes unstable upon feedback path changes (phone to ear), witnessed here at $0.5 \mathrm{~s}, 3.1 \mathrm{~s}$, and $6.8 \mathrm{~s}$, indicated by feedback distortions in the frequency region of $2-3 \mathrm{kHz}$.

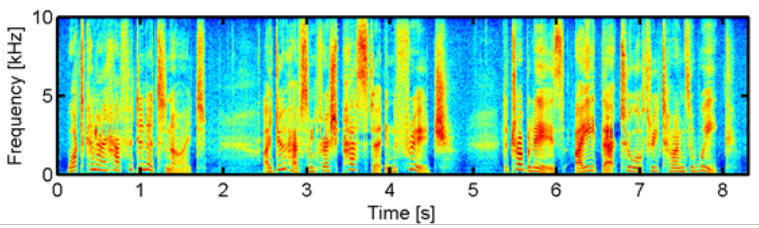

Figure $\mathbf{5}$ This spectrogram shows the feedback control system with the STM based technology. The system remains stable upon feedback path changes (phone to ear) after $0.5 \mathrm{~s}, 3.1 \mathrm{~s}$, and $6.8 \mathrm{~s}$, and the STM processed sounds replace the feedback distortions. The STM is only active in time-frequency regions where the traditional system cannot efficiently remove feedback. In this way, soft and non-intrusive STM processed sounds replace traditional loud and annoying feedback distortions.

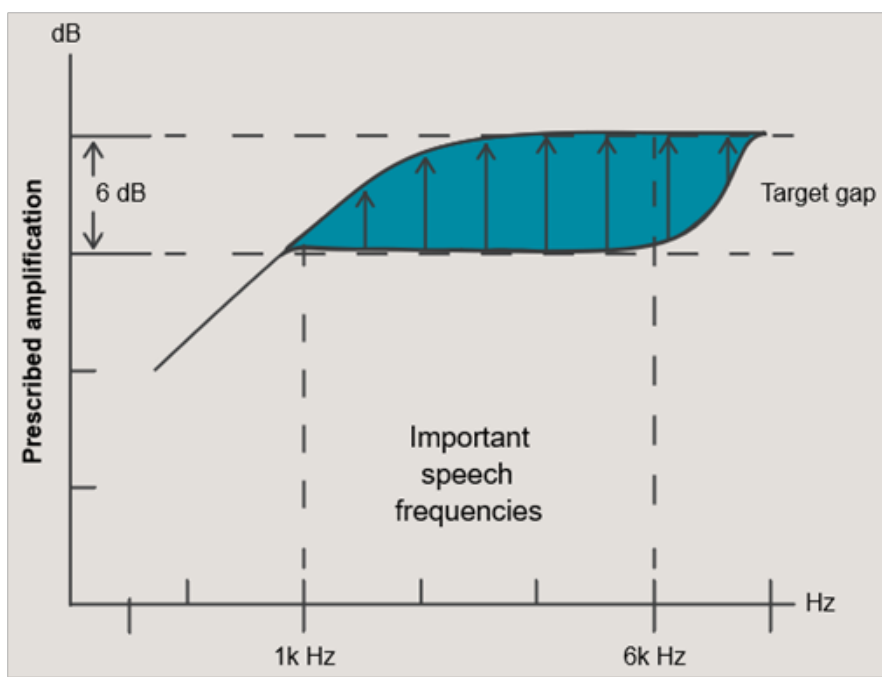

Figure 6 Demonstrates the difference between the prescribed target gain versus actual gain achieved. That is, due to the risk of feedback, the prescribed gain in hearing aids is often attenuated (i.e., not achieved) to prevent audible feedback. This unfortunate gain reduction often occurs in the most important high frequency speech frequencies, as feedback in hearing aids is typically dominant in the frequency range of $2-5 \mathrm{kHz}$ (Hellgren et al. 1999).$^{50}$ 

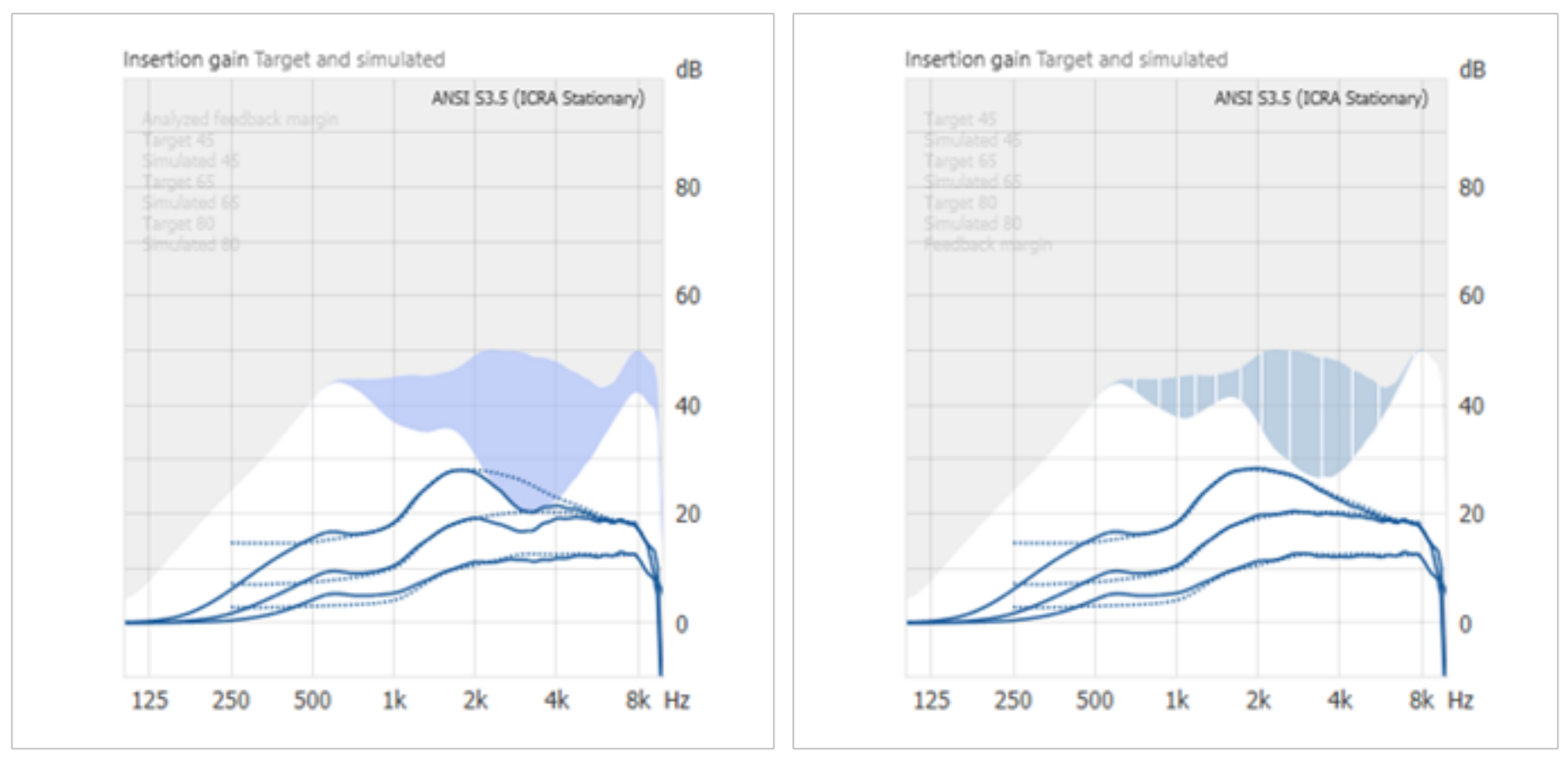

Figure $7 \mathrm{~A}$ traditional feedback control system which reduces gain in dynamic feedback situations (left graph) whereas the new feedback control system (Opn S) maintains the prescribed gain without feedback (right graph). The dotted/solid lines show the prescriptive targets/prescribed gains, at soft/moderate/loud sound levels. The prescribed gains are potentially lower than the prescriptive targets due to the risk of feedback indicated by the blue shaded areas.

\section{Conclusions}

For many people, hearing and listening are synonymous. For the learned HCP, they are quite different. After we have comprehensively evaluated a specific patient, we can then attribute their auditory problem(s) appropriately to hearing, listening and/or other problems. Again, diagnosis first, treatment second. Importantly, when listening problems are identified, the single most significant rehabilitative factor (after accomplishing audibility) is improving the SNR. Of note, adjusting the loudness controls (such as gain and compression ratios) in isolation on a generic hearing aid are not likely to improve the SNR. Admittedly, because patients confuse hearing and listening, they are likely to perceive and interpret louder sounds as an improvement. However, that percept is likely to prove faulty when patients listen to SIN in challenging acoustic environments such as restaurants and cocktail parties. Improving the SNR is best accomplished via knowledgeable and learned HCPs, using contemporary fitting formulas and advanced algorithms which allow advanced hearing aids to achieve prescriptive targets while improving the SNR, without acoustic feedback. When these technologies and protocols are combined with Best Practice verification and validation procedures, likely outcomes include; a decrease in returned instruments, an increase in perceived sound quality, increased patient satisfaction and an improved ability to understand SIN.

\section{Acknowledgments}

None.

\section{Conflict of interests}

The authors declare that there is no conflict of interests regarding the publication of this paper.

\section{Funding}

None.

\section{References}

1. Rosenblum G, Beck DL. Over The Counter Hearing Aids. In The Hearing Professional, published by the International Hearing Society, 2018.

2. Beck DL, Danhauer JL. Amplification for adults with hearing difficulty, speech in noise problems - and normal thresholds. Journal of Otolaryngology-ENT Research. 2019;11(1).

3. Beck DL, Danhauer JL, Abrams HB, et al. Audiologic considerations for people with normal hearing sensitivity yet hearing difficulty and/or speech-in-noise problems. Hearing Review. 2018;25(10):28-38.

4. Fay RR. Hearing in Vertabrates: A Psychoacoustic Databook. Hill-Fay Associates. Winetka: Illinois; 1988.

5. Beck, DL, Flexer, C. Listening is Where hearing Meets Brain in Adults and Children. 2011.

6. Timmer BHB, Hickson L, Launer S. Adults with Mild Hearing Impairment - Are We Meeting the Challenge? Int J Audiol. 2015;54(11):786-795.

7. Shub, Makashay, Brungart. Predicting Speech-in-Noise Deficits from the Audiogram. Ear \& Hearing. 2019.

8. Goyette A, Crukley J, Galster J. The Effects of Varying Directional Bandwidth in Hearing Aid Users' Preference and Speech-in-Noise Performance. Am J Audiol. 2018;8;27(1):95-103.

9. American Speech Language Hearing Association. Preferred Practice Patterns for the Profession of Audiology. ASHA. 2006.

10. American Academy of Audiology. Guidelines for the Audiological Management of Adult Hearing Impairment. Audiology Today. 2006;18(5):32-37. 
11. British Society of Audiology (BSA). Guidance on the use of real ear measurements to verify the fitting of digital signal processing hearing aids. 2007.

12. International Hearing Society (HIS) Best Practice Guidelines. 2019.

13. Killion MC. New thinking on hearing in noise: a generalized articulation index. Sem Hear. 2002;23(1):57-75.

14. Dillon H, Ching T, Golding M. Hearing Aids for Infants and Children. In Pediatric Audiology: Diagnosis, Technology and Management. 2nd ed. Madell \& Flexer. Thieme Publishers; 2011.

15. Wilson RH. Development of a speech-in-multi-talker babble paradigm to assess word-recognition performance. $J$ Am Acad Audiology. 2003;14(9):453-470.

16. Bamford J, Kowal A, Bench J. The BKB (Bamford-Kowal-Bench) Sentence Lists for partially-hearing children. Br Journal of Audiology. 1979;13(3):1-8-112.

17. Beck DL, Benitez L. A Two-Minute Speech-In-Noise Test: Protocol and Pilot Data. Audiology Today. 2019.

18. Scollie S, Seewald R, Cornelisse L, et al. The Desired Sensation Leve multistage input/output algorithm. Trends Amplif. 2005;9(4):159-197.

19. Keidser G, Dillon H, Flax M, et al. The NAL-NL2 prescription procedure. Audiology Research. 2011;1(1):88-90.

20. Jorgensen L. Verification and Validation of Hearing Aids: Opportunity Not an Obstacle. Journal of Otology. 2016;11(2):57-62.

21. Bentler R, Mueller HG, Ricketts TA. Probe microphone clinical uses. In Bentler, Mueller, Ricketts, editors. Modern Hearing Aids: Verification, outcome measures, and follow-up. San Diego, CA: Plural Publishing, Inc. 2016. p. 283-347.

22. Johnson EE, Dillon H. A comparison of gain for adults from generic hearing aid prescriptive methods: impacts on predicted loudness, frequency bandwidth, and speech intelligibility. $J$ Am Acad Audiol. 2011:22(7):441-459.

23. Keidser G, Alamudi K. Real-life efficacy and reliability of training a hearing aid. Ear Hear. 2013;34(5):619-629.

24. Mueller HG, Hornsby BWY. Trainable hearing aids: the influence of previous use-gain. Audiology Online. 2014.

25. Polonenko MJ, Scollie S, Moodie S, et al. Fit to targets, preferred listening levels, and self-reported outcomes for the DSL v5.0a hearing aid prescription for adults. Int $J$ Audiol. 2010;49(8):550-560.

26. Abrams HB, Chisolm TH, McManus M, et al. Initial-fit approach versus verified prescription: comparing self-perceived hearing aid benefit. $\mathrm{J} \mathrm{Am}$ Acad Audiol. 2012;23(10):768-778.

27. Valente M, Odeing K, Brockmeyer A, et al. Differences in Word and Phoneme Recognition in Quiet, Sentence Recognition in Noise, an Subjective Outcomes between Manufacturer First-Fit and Hearing Aids Programmed to NAL-NL2 Using Real-Ear Measures. J Am Acad Audiol. 2017.

28. Folkeard P, Pumford J, Abbasalipour P, et al. A comparison of automated real-ear and traditional hearing aid fitting methods. Hearing Review. 2018;25(11):28-32.

29. Folkeard P, Pumford J, Pietrobon J, et al. Validation of the Audioscan Verifit2 Probe Tube Placement Tool. Paper presented at the American Academy of Audiology Conference, Columbus, OH, 2019.

30. Pumford J, Sinclair S. Real-ear measurement: Basic terminology and procedures. Audiology Online. 2001.
31. Pumford J, Smriga D. Fundamentals of Real-Ear Measurement (Chapter 7). In Galster J, editor. Audiology Treatment 3rd ed. Thieme: New York; 2018. p. 114-151.

32. Aazh H, Moore BC. The value of routine real ear measurement of the gain of digital hearing aids. J Am Acad Audiol. 2007;18(8):653-664.

33. Beck DL, Duffey J. Visible Speech: A Patient-Centered Clinical Tool Hearing review. 2007.

34. Aazh H, Moore BC, Prasher D. The accuracy of matching target insertion gains with open-fit hearing aids. Am J Audiol. 2012;21(2):175-180.

35. Sanders J, Stoody T, Weber J, et al. Manufacturers' NAL-NL2 Fittings Fail Real-ear Verification. Hearing Review. 2015;21(3):24.

36. Amlani AM, Pumford J, Gessling E. Real-ear measurement and its impact on aided audibility and patient loyalty. Hearing Review. 2017;24(10):1221.

37. Leavitt R, Flexer C. The importance of audibility in successfu amplification of hearing loss. Hear Review. 2012;19(13):20-23.

38. Kochkin S. MarkeTrak VIII. Reducing patient visits through verification and validation. Hearing Review. 2011;18(6):10-12.

39. Amlani AM, Pumford J, Gessling E. Improving Patient Perception of Clinical Services Through Real-ear Measurements. Hearing Review. 2016;23(12):12.

40. Spriet A, Rombouts G, Moonen M, et al. Adaptive feedback cancellation in hearing aids. Elsevier J Franklin Inst. 2006;343(6):545-573.

41. Van Waterschoot T, Moonen M. Adaptive feedback cancellation for audio applications. Elsevier Signal Process. 2009;89(11):2185-2201.

42. Guo M, Jensen SH, Jensen J. Novel acoustic feedback cancellation approaches in hearing aid applications using probe noise and probe noise enhancements. IEEE Trans Audio Speech Lang Process. 2012;20(9):25492563

43. Nakagawa CRC, Nordholm, SF Albu, et al. Closed-loop feedback cancellation utilizing two microphones and transform domain processing. IEEE. 2014. p. 3673-3677.

44. Schepker H, Tran LTT, Nordholm S, et al. Acoustic feedback cancellation for a multi-microphone earpiece based on a null-steering beamformer. In Proc. 15th Int. Workshop Acoust Signal Enhancement. 2016. p. 1-5.

45. Klinkby KT, Noergaard PM, Pontoppidan Föh H. Hearing aid and method of estimating dynamic gain limitation in a hearing aid. International Patent WO/2007/112777, 2006.

46. Fretz RJ. Hearing aid circuit with feedback transition adjustment. US Patent 8355517B1, 2010.

47. Guo M, Kuenzle B. On the use of spectro-temporal modulation in assisting adaptive feedback cancellation for hearing aid applications. In Proc 51st Asilomar Conf Signals Syst Comput. 2017.

48. Guo M, Kuriger M, Lesimple C, et al. Extension and evaluation of a spectro-temporal modulation method to improve acoustic feedback performance in hearing aids. In Proc. 2018 IEEE Int Conf Acoust Speech Signal Process. 2018; 246-250.

49. Callaway SL. Introduction to Open Sound Optimizer, Oticon Whitepaper, 2018 .

50. Hellgren, Lunner, Arlinger. Variations in the feedback of hearing aids. $J$ Acoust Soc Amer. 1999;106(5):2821-2833.

51. Jensen JJ. Oticon Opn S Clinical Evidence. Oticon Whitepaper, 2018.

52. Dillon H. Hearing Aids. Stuttgart, Thieme: Germany; 2001. 